\title{
The glutaredoxin ATGRXS13 is required to facilitate Botrytis cinerea infection of Arabidopsis thaliana plants
}

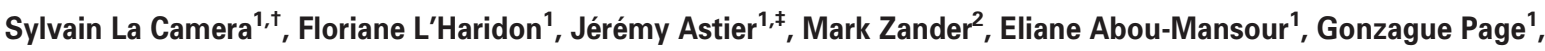 \\ Corinna Thurow ${ }^{2}$, David Wendehenne ${ }^{3}$, Christiane Gatz ${ }^{2}$, Jean-Pierre Métraux ${ }^{1}$ and Olivier Lamotte ${ }^{1,3, *}$ \\ ${ }^{1}$ Département de Biologie, Université de Fribourg, 10 chemin du Musée, CH-1700 Fribourg, \\ ${ }^{2}$ Albrecht-von-Haller-Institut für Pflanzenwissenschaften, Georg-August-Universität Göttingen, Untere Karspüle 2, D-37073 \\ Göttingen, Germany, and \\ ${ }^{3}$ UMR CNRS 5184 INRA 1088 Université de Bourgogne, Plante-microbe-environnement, 17 rue Sully, BP 86510, F-21065 Dijon \\ Cedex, France
}

\begin{abstract}
*For correspondence (fax +33 38069 3753; e-mail olivier.lamotte@dijon.inra.fr).
†Present address: UMR CNRS 6503 LACCO, Laboratoire de Catalyse en Chimie Organique, Equipe "Physiologie Moléculaire du Transport des Sucres chez les végétaux." 40, Avenue du Recteur Pineau. F-86022 Poitiers cedex, France.

${ }^{\ddagger}$ Present address: UMR CNRS 5184 INRA1088 Université de Bourgogne, Plante-microbe-environnement, 17 rue Sully, BP 86510, F-21065 Dijon Cedex, France.
\end{abstract}

\begin{abstract}
SUMMARY
Botrytis cinerea is a major pre- and post-harvest necrotrophic pathogen with a broad host range that causes substantial crop losses. The plant hormone jasmonic acid (JA) is involved in the basal resistance against this fungus. Despite basal resistance, virulent strains of $B$. cinerea can cause disease on Arabidopsis thaliana and virulent pathogens can interfere with the metabolism of the host in a way to facilitate infection of the plant. However, plant genes that are required by the pathogen for infection remain poorly described. To find such genes, we have compared the changes in gene expression induced in $A$. thaliana by JA with those induced after $B$. cinerea using genome-wide microarrays. We have identified genes that are repressed by JA but that are induced by $B$. cinerea. In this study, we describe one candidate gene, ATGRXS13, that encodes for a putative glutaredoxin and that exhibits such a crossed expression. In plants that are infected by this necrotrophic fungus, ATGRXS13 expression was negatively controlled by JA and TGA transcription factors but also through a JA-salicylic acid (SA) cross-talk mechanism as $B$. cinerea induced SA production that positively controlled ATGRXS13 expression. Furthermore, plants impaired in ATGRXS13 exhibited resistance to $B$. cinerea. Finally, we present a model whereby $B$. cinerea takes advantage of defence signalling pathways of the plant to help the colonization of its host.
\end{abstract}

Keywords: glutaredoxin, Botrytis cinerea, jasmonic acid, salicylic acid, necrotrophic pathogen.

\section{INTRODUCTION}

Resistance of plants to pathogens is a combination of constitutive barriers and inducible defence reactions that can be induced after an encounter with a potential pathogen. They include production of antimicrobial metabolites and proteins, histological barriers or the induction of a cell-death response. This innate immunity is activated upon recognition of pathogen- or microbe-associated molecular patterns (PAMPs or MAMPs) or upon recognition of damage inflicted by insects or wounds (damage-associated molecular patterns; DAMPs) by corresponding plant receptors. This is rapidly followed by changes in ion fluxes, production of reactive oxygen species and nitric oxide, and by the activation of protein phosphorylation involving mitogen-activated protein (MAP) kinase cascades, finally leading to localized defence responses (Boller and Felix, 2009; Nürnberger and Kemmerling, 2009). Resistance can also be induced in tissues remotely located from the infection site (Vlot et al., 2008) and comprises systemic acquired resistance (SAR) in which resistance is induced by a pathogen infection on a lower leaf (Durrant and Dong, 2004) and induced systemic resistance (ISR) triggered by beneficial root-associated microorganisms (Van Loon et al., 1998). 
An ongoing question concerns the understanding how virulent pathogens cope with such a sophisticated defence system. Avoidance and tolerance of antimicrobials constitutes an important mechanism displayed by virulent pathogens (Van Etten et al., 2001). Interference with host defence by a pathogenic effector was long postulated before its existence was demonstrated experimentally. Effectors produced by virulent pathogens can directly interact and inhibit host defences. Their actions have been described for specialized hemibiotrophic or biotrophic bacterial, oomycete pathogens (Chisholm et al., 2006; da Cunha et al., 2007) or nematodes (Davis et al., 2008). New insights start to gain understanding in the specific interactions of plants with fungi (Métraux et al., 2009) but to date little information is known about the action of effectors of fungal pathogens characterized by a broad host range.

The signalling for innate immunity is relayed by hormones such as SA, JA or ethylene (ET) and leads to changes in gene expression that are associated with enhanced resistance (Bari and Jones, 2009). For instance, JA is generally associated with basal levels of defence against the necrotrophic fungus $B$. cinerea in $A$. thaliana (Glazebrook, 2005). However, some classical studies have shown how pathogens can also use the power of hormones to their advantage. Examples include the infections by Gibberella fujikuroi, Agrobacterium tumefaciens or Pseudomonas savastanoi that all result in hormonal imbalances with consequences of uncontrolled growth of the invaded tissue favoring pathogen invasion (Robert-Seilaniantz et al., 2007). These observations provide the conceptual basis for the experimental approach used here to find host genes that are required by virulent pathogens such as the necrotrophic fungus Botrytis cinerea to infect plants.

To find such plant genes we compared the changes in gene expression in $A$. thaliana induced by JA with those induced by $B$. cinerea using genome-wide microarrays and have identified a group of genes that exhibit a crossed expression pattern: they are repressed by JA but induced by $B$. cinerea. We hypothesized that this expression would be indicative for genes whose expression is altered by $B$. cinerea to its advantage. On one hand, the increased expression of such genes after infection by $B$. cinerea could therefore help the pathogen in its colonization of the host. On the other hand, JA-repressed genes might be part of a protective reaction of the plant towards $B$. cinerea. Thus, a JA-dependent signalling pathway might repress plant genes required for susceptibility to B. cinerea. We would predict that inactivation of such genes should alter the interaction of $B$. cinerea with the plant. We describe one candidate gene, ATGRXS13, obtained from such a search for crossed expression. The expression of ATGRXS13 that encodes a putative glutaredoxin is repressed by JA and induced by $B$. cinerea. Plants impaired in ATGRXS13 expression exhibit partial resistance to $B$. cinerea. We present a model whereby
B. cinerea takes advantage of defence signalling pathways of the plant to help its colonization of the host.

\section{RESULTS}

\section{Botrytis cinerea strain BMM induces SA and JA production in Arabidopsis plants}

After inoculation of wild-type (WT) Arabidopsis with B. cinerea strain BMM, the concentration of SA (both free and conjugated) increased from 0.31 to $0.78 \mu \mathrm{g} \mathrm{g}^{-1}$ of fresh weight (FW) at 36 hours post inoculation (hpi) compared with uninfected controls in which the SA level remained low and stable from 0 to $48 \mathrm{hpi}$. In the dde2-2 mutant defective in JA biosynthesis (von Malek et al., 2002), an increase in SA concentration was already detected after $24 \mathrm{hpi}$ and reached $3.8 \mu \mathrm{g} \mathrm{g}^{-1} \mathrm{FW}$ at $48 \mathrm{hpi}$ in comparison with $0.7 \mu \mathrm{g} \mathrm{g}^{-1} \mathrm{FW}$ in the WT at the same time-point (Figure 1a). Accordingly, the SA marker gene PR1 was more expressed in dde2-2 in comparison with WT plants after infection (Figure S1a). The SA level in the SA defective sid2-1 mutants (Nawrath and Métraux, 1999) remained low and unchanged after B. cinerea inoculation (Figure 1a).

An increase in JA accumulation was observed at $36 \mathrm{hpi}$ in WT $\left(0.08 \mu \mathrm{g} \mathrm{g}^{-1} \mathrm{FW}\right)$ and sid2-1 plants $\left(0.05 \mu \mathrm{g} \mathrm{g}^{-1} \mathrm{FW}\right)$ whereas JA was not detected in mock-inoculated plants. At $48 \mathrm{hpi}$, the level of JA was similar in WT $\left(0.34 \mu \mathrm{g} \mathrm{g}^{-1} \mathrm{FW}\right)$ and in sid2-1 plants $\left(0.37 \mu \mathrm{g} \mathrm{g}^{-1} \mathrm{FW}\right)$. However, JA was not detected in dde2-2 after inoculation with $B$. cinerea (Figure 1b).

These results show that the inoculation with $B$. cinerea is followed by both an increase in SA and JA at 36 hpi and highlight the JA/SA crosstalk; mutants impaired in JA synthesis accumulated more SA and expressed more $P R 1$ after $B$. cinerea inoculation.

\section{AtGRXS13 is induced and alternatively spliced after B. cinerea infection}

Our goal was to identify $A$. thaliana genes that are required for the colonization by a virulent pathogen. We have used a simple concept of crossed expression to find potential candidates. As basal resistance to the virulent fungus B. cinerea depends on JA (Ferrari et al., 2003; Rowe et al., 2010 ) and because JA is induced upon infection by $B$. cinerea, we have used microarray data available on public databases to compile genes whose expression is changed after inoculation with $B$. cinerea and treatment with JA using the Excel macro FiRe (Garcion et al., 2006). As expected, the expression of most of the genes either increased or decreased after $B$. cinerea or JA. Interestingly, a small group of genes departed clearly from this pattern and showed an increase in expression after $B$. cinerea inoculation but also decrease in expression after JA treatment. We have focused on one such gene, ATGRXS13 (AT1G03850), encoding a putative glutaredoxin. Glutaredoxins are involved in redox 

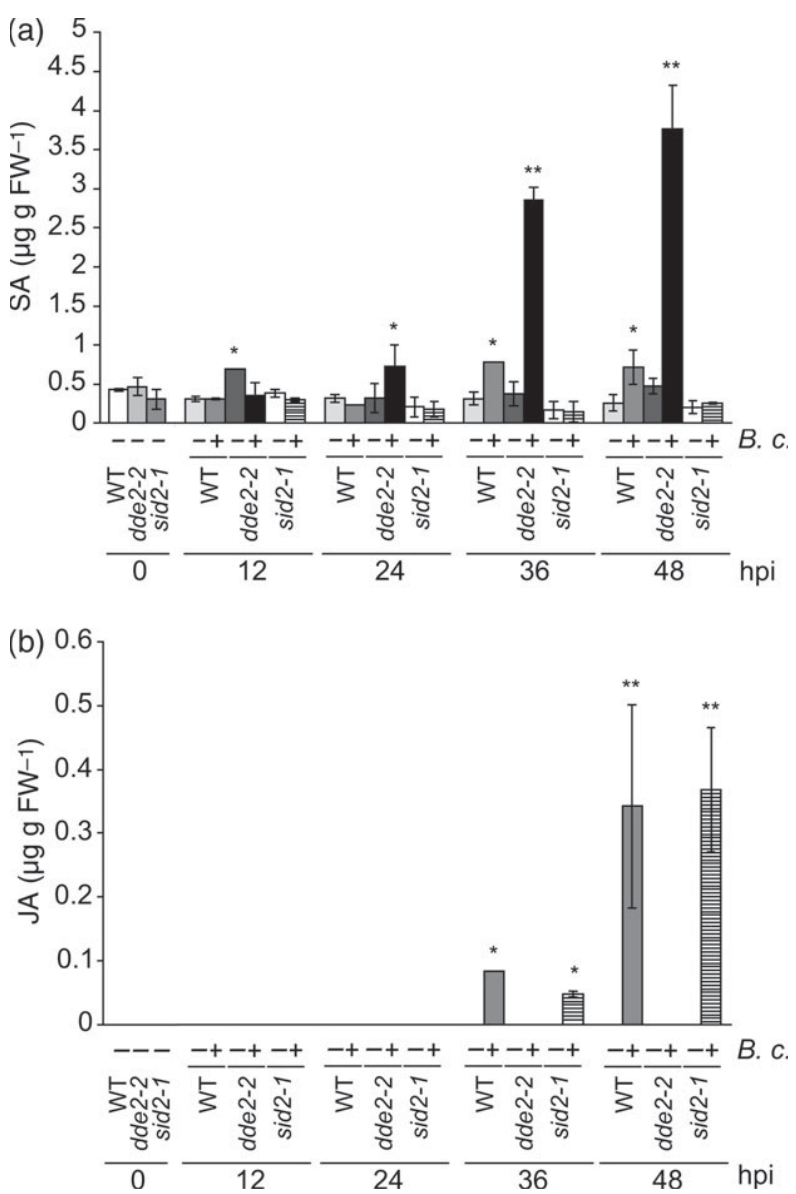

Figure 1. Accumulation of SA and JA in WT Arabidopsis plants and mutants infected with $B$. cinerea.

Total salicylic acid (SA) (a) and jasmonic acid (JA) (b) accumulation in wild type (WT), dde2-2 and sid2-1 mutants. All plants were sprayed at time 0 with the mock buffer or a spore suspension of $B$. cinerea. Two replicates of leaves from at least four plants were collected at various time points after treatment. The data represent the mean of two independent experiments $( \pm S D)$. No star or different stars represent groups which were significantly different from one another as determined by a one-way analysis of variance (ANOVA) followed by a multiple comparison with the Student-Newman-Keuls method $(P<0.05)$. hpi: hour post inoculation. B. c.: Botrytis cinerea.

regulation of proteins (Rouhier et al., 2004). As B. cinerea infection is accompanied by oxidative stress, we suspected that this protein might be a target for altered regulation upon $B$. cinerea infection.

We have isolated three mRNA corresponding to ATGRXS13 indicating that ATGRXS13 was alternatively spliced in $A$. thaliana plants infected with $B$. cinerea. Only two transcripts, AT1G03850.1 and AT1G03850.2, are described in the TAIR (The Arabidopsis Information Resource) database. An alignment of the three transcript sequences and the genomic sequence of ATGRXS13 is shown (Figures $\mathrm{S} 2$ and 2a). AT1G03850.2 is composed of a unique exon and is predicted to encode a protein of 150 amino acid residues (Figure 2). However, AT1G03850.1 and
AT1G03850.3 are composed of two exons surrounding one intron (Figure 2a). They are predicted to encode two proteins of respectively 159 and 158 amino acid residues (Figure 2b). AT1G03850.3 is produced by the use of an alternative splice donor site in the first exon. This splicing event causes a frame-shift that generates a different stop codon leading a different $\mathrm{C}$-terminal of the protein (Figures $2 \mathrm{~b}$ and $\mathrm{S} 2$ ). Although AT1G03850 is alternatively spliced, the three predicted AT1G03850 proteins maintained the glutaredoxin putative catalytic site CCLG (Figure 2b). The characteristic amino acid sequence that defines a GRX domain (Couturier et al., 2009) was only found in AT1G03850.1 and AT1G03850.2. More precisely, the glycyl residue located at position 124 in the putative glutathione binding site was absent in AT1G03850.3. Furthermore, the prediction of the secondary structures of the proteins from primary amino acid sequence indicated that only AT1G03850.1 and AT1G03850.2 possess a secondary structure typical of a thioredoxin fold, a characteristic feature of GRX (Martin, 1995). Finally, the ALWL motif present in the C-terminal end of many GRX from the group III (Li et al., 2009) was only present in isoform 2 (Figure 2b). The induction of expression of each splice variant after B. cinerea inoculation was confirmed by quantitative reverse transcription polymerase chain reaction (qRT-PCR) using specific primers (Table S1) and was normalized against a constitutive gene as described by Czechowski et al. (2005). Primers used to detect AT1G03850 splice variants have the same PCR efficiency (data not shown). In non-treated and mock-treated WT plants, each splice variant was expressed at low basal levels, but the transcripts accumulated within $24 \mathrm{~h}$ after infection with B. cinerea (Figure 3). Interestingly, by comparing the normalized expression values of AT1G03850 splice variants, it can be seen that $A T 1 G 03850.2$ was much more abundant than AT1G03850.3 and AT1G03850.1 (Figure 3).

\section{Mutation in ATGRXS13 alters the susceptibility of}

\section{$A$. thaliana to $B$. cinerea}

To determine the biological importance of ATGRXS13, mutants homozygous for T-DNA insertions (Alonso et al., 2003) in ATGRXS13 were identified by PCR and the genomic DNA surrounding each T-DNA insertion was sequenced to precisely localize the insertions (Figure 2a). A residual expression of ATGRXS13 splice variants was observed in grxs13-1 following mock and $B$. cinerea treatment but ATGRXS13 was no longer inducible after $B$. cinerea inoculation (Figure 4). These results can be explained by the localization of the T-DNA-insertion in the promoter region of the gene. In grxs13-2, the expression of ATGRXS13 transcripts was barely detectable.

To test the susceptibility of grxs 13 mutants to B. cinerea, plants were drop-inoculated with a solution of conidia of B. cinerea and lesion diameters (LD) were measured after 

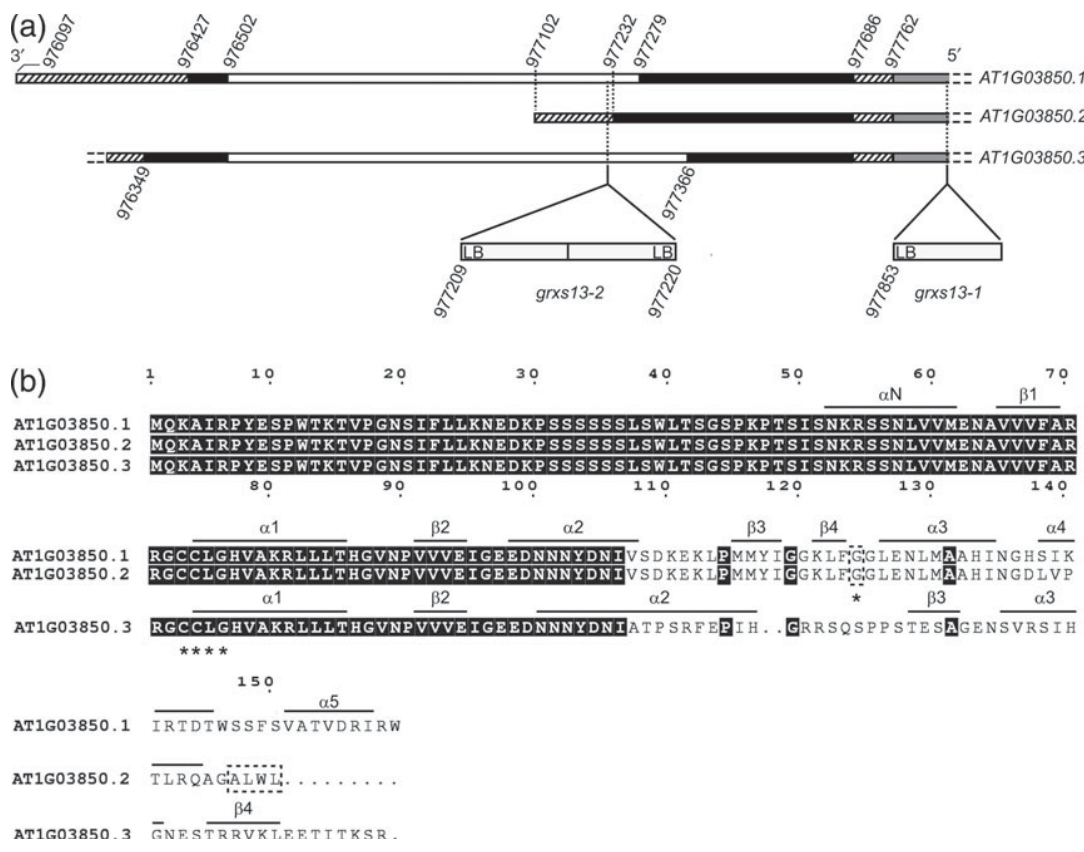

Figure 2. Structure of the ATGRXS13 gene and protein.

(a) Schematic representation of ATGRXS13 splice variants. Slanted numbers indicate the coordinates of AT1G03850 splice variants on the chromosome 1 (http:// www.arabidopsis.org). The positions of T-DNA insertions in grxs13 mutants are displayed. Grey: promoter; hatching: $5^{\prime}$ - and $3^{\prime}$-untranslated region (UTR); black: exon; white: intron, LB: left border of T-DNA.

(b) Alignment of the deduced amino acid sequence from the three AT1G03850 splice variants. Sequences were aligned with ClustalW (Thompson et al., 1994) and displayed using ESPript (Gouet et al., 2003). Secondary structures were predicted using Psi-Pred (Jones, 1999). Stars indicate the conserved residue CCLG in the putative active site at position 73-76 and the putative glutathione (GSH)-binding site at position 124 (dashed box and star). The ALWL motif specific to the second isoform is surrounded by a dashed box.

3 days. WT plants were susceptible to the $B$. cinerea strain BMM (Figures $5 a$ and S3). A decrease in LD in grxs13 mutants was observed indicating that both mutants were more resistant to $B$. cinerea than WT plants (Figure 5a). More small (LD $<2 \mathrm{~mm}$ ) and less large ( $L D>6 \mathrm{~mm}$ ) lesions were observed in the two grxs 13 mutants in comparison with WT plants (Figure S3). To confirm this result, we quantified fungal growth in both WT and grxs 13 mutants. Fungal growth was reduced at $48 \mathrm{hpi}$ in both grxs 13-1 and grxs 13-2 mutants in comparison with WT plants (Figure 5b). These results indicate that $A T G R X S 13$ is required to facilitate $B$. cinerea infection of $A$. thaliana plants.

As three ATGRXS13 mRNA accumulate during B. cinerea infection (Figure 3), the role of each ATGRXS13 isoform was tested separately during infection. To this end, the cDNAs corresponding to each of the three ATGRXS13 mRNA were introduced into grxs 13-1 under the control of $1500 \mathrm{bp}$ of the native promoter of ATGRXS13 (Figure 5c). AT1G03850.2 was able to complement grxs13-1 resistance phenotype whereas AT1G03850.1 and AT1G03850.3 were not (Figure $5 \mathrm{c}$ ). Furthermore, transgenic plants constitutively expressing ATGRXS13 splice variants were generated. The cDNA corresponding to each ATGRXS13 mRNA were placed under the control of the CaMV35S promoter. Over-expression of $A T 1 G 03850.2$ in WT plants resulted in an increased susceptibility to $B$. cinerea, whereas over-expression of AT1G03850.1 and AT1G03850.3 had no effect (Figure 5d). $A$ recent study has indicated that over-expression of ATGRXC7/ROXY1 in Arabidopsis results in a strong accumulation of reactive oxygen species (ROS) in transformed plants (Wang et al., 2009) that correlates with an enhanced susceptibility to $B$. cinerea of these plants. By contrast, plants over-expressing each GRXS13 splice variants do not accumulate more ROS (Figure S4). In conclusion, among the three isoforms of ATGRXS13, only AT1G03850.2 is likely to be required for infection of Arabidopsis by $B$. cinerea. Thus, we focused our attention on the role of AT1G03850.2 in this context.

Induction of AtGRXS13 expression after $B$. cinerea infection depends on both SA and JA signalling pathways

We determined the contribution of signalling pathways commonly associated with plant defence responses to the induction of ATGRXS13 (Figure 6). We tested mutants defective either in the SA (sid2-1, eds5-3; Heck et al., 2003; Nawrath and Métraux, 1999) or in the JA pathway (dde2-2, opr3, coi1-16; Stintzi and Browse, 2000; von Malek et al., 2002; Xie et al., 1998) and the npr1-1 mutant, a key positive regulator of SA-mediated gene expression (Spoel et al., 2003). After B. cinerea infection, the accumulation of 

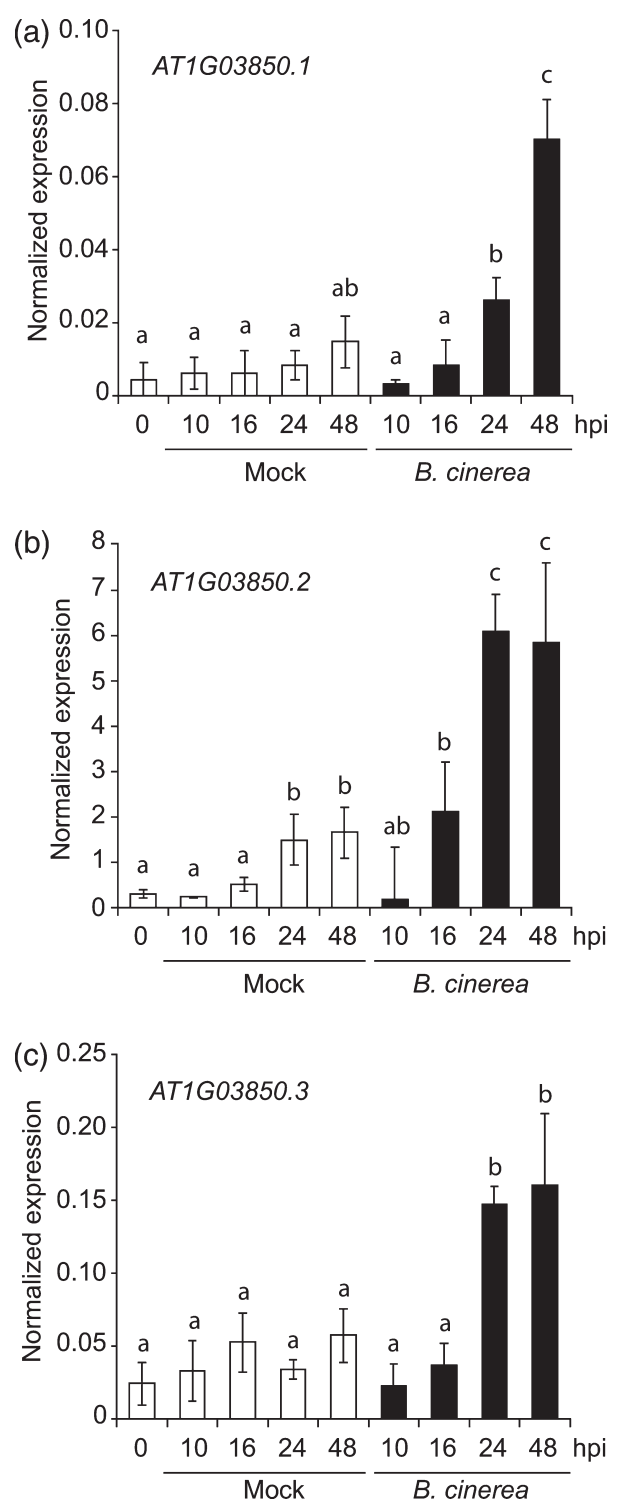

Figure 3. ATGRXS13 mRNAs accumulation in wild-type (WT) Arabidopsis plants infected with $B$. cinerea.

WT Col plants were sprayed with mock solution (white bars) or $B$. cinerea spores (black bars). Leaves from four to six plants were harvested at different time points for each treatment. AT1G03850.1 (a), AT1G03850.2 (b), $A T 1 G 03850.3$ (c) transcripts levels were quantified by real-time polymerase chain reaction (PCR). Data are expressed as normalized expression (no unit) to the plant reference gene AT4G26410 transcript level (Czechowski et al., 2005) and represent the mean of four independent experiments $( \pm S D)$. Different letters represent groups which were significantly different from one another as determined by a one-way ANOVA followed by a multiple comparison with the Student-Newman-Keuls method $(P<0.05)$.

AT1G03850.2 mRNA was strongly increased in JA-related mutants but was strongly impaired in SA-related mutants in comparison with WT plants (Figure 6).

Furthermore, ATGRXS13 was induced after exogenous treatment of Arabidopsis plants with $1 \mathrm{~mm}$ of SA at all time points analyzed confirming that this gene is positively (a)

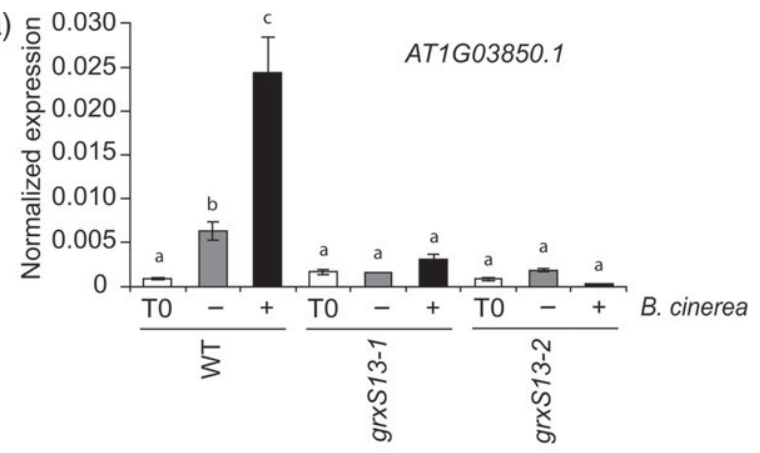

(b)

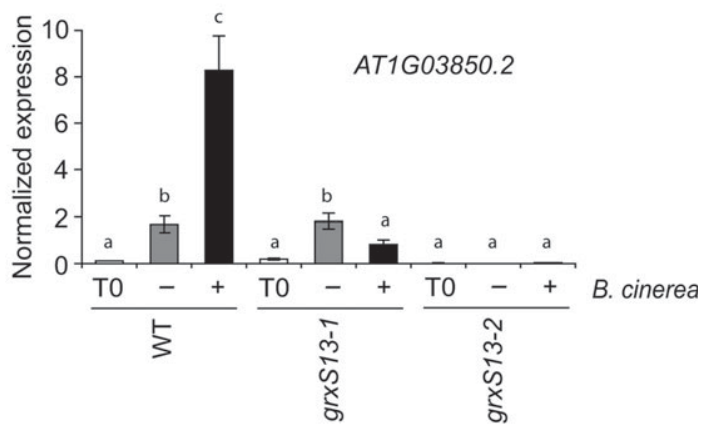

(c)

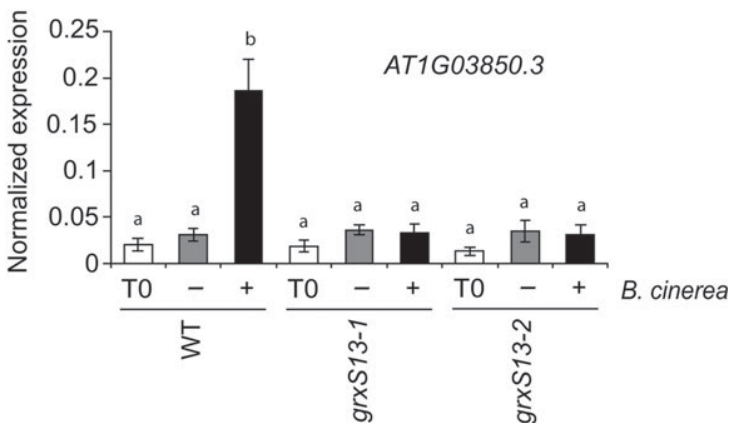

Figure 4. ATGRXS13 mRNAs accumulation in WT and grxs13 mutants infected with $B$. cinerea.

The leaves from six wild-type (WT) Col and grxs13 plants were not sprayed (white bars), sprayed with mock solution (grey bars) or B. cinerea spores (black bars) and were harvested after 24 h. AT1G03850.1 (a), AT1G03850.2 (b), AT1G03850.3 (c) transcript levels were quantified by real-time PCR and expressed as normalized expression (no unit). The data represent the mean of four independent experiments $( \pm S D)$. Different letters represent groups that were significantly different from one another as determined by a one-way analysis of variance (ANOVA) followed by a multiple comparison with the Student-Newman-Keuls method $(P<0.05)$.

regulated by SA (Figure 7a). The JA-related mutants are more susceptible to $B$. cinerea than WT plants (Figure S1b; Ferrari et al., 2003). To exclude the possibility that the overinduction of ATGRXS13 in these mutants is the consequence of a stronger infection, we tested whether JA could negatively regulate the induction of $A T G R X S 13$ by SA. We sprayed plants with $100 \mu \mathrm{M}$ of MeJA $3 \mathrm{~h}$ before application of SA and analyzed ATGRXS13 gene induction $6 \mathrm{~h}$ after SA treatment. MeJA pretreatment reduced the induction of ATGRXS13 by SA indicating that JA negatively controls ATGRXS13 expression (Figure 7b). 
According to these results, we can conclude that JA and SA regulate the induction of ATGRXS13 in opposing ways after inoculation by $B$. cinerea.

\section{GRXS13 is negatively regulated by TGA transcription factors that also bind to GRXS13 protein}

TGA2 clade of transcription factors has been shown to be mediator of plant defence in Arabidopsis plants challenged with B. cinerea (Zander et al., 2010). We identified the TGACGTCA sequence in the promoter of AT1G03850 corresponding to the perfect binding site for TGA transcription factor (Figure S5; Qin et al., 1994). Furthermore, the TGACG sequence sufficient for TGA recognition (Spoel et al., 2003) was found eight times within this promoter. We tested the hypothesis that this class of TGA transcription factors modulates ATGRXS13 expression after $B$. cinerea infection. The expression of $A T G R X S 13.2$ was stronger in the tga2tga5tga6 triple mutant in comparison with WT plants indicating that the TGA2 clade of transcription factors negatively regulates ATGRXS13 gene expression during infection with $B$. cinerea (Figure 8a). Members of the Arabidopsis group III GRX can directly interact with TGA transcription factors (Ndamukong et al., 2007; Li et al., 2009). We tested whether this can also be true for ATGRXS13 and TGA2 in a yeast two-hybrid assay. The $\beta$-galactosidase activity was induced only when both ATGRXS13 and TGA2 proteins were introduced in yeast cells indicating that both proteins interact (Figure 8b).

\section{Classical defence genes are not controlled by AtGRXS13}

To explain the resistance phenotype of grxs 13 mutants, we tested whether the expression of the JA marker PDF1.2 and the SA marker PR1 were affected in grxs13-1 after infection by B. cinerea. PDF1.2 was induced at $24 \mathrm{hpi}$ (Figure S6b) whereas PR1 was strongly induced $48 \mathrm{hpi}$ (Figure S6a) as previously described (Chassot et al., 2007). However, neither PDF1.2 nor PR1 induction were impaired in grxs 13-1 mutant after $B$. cinerea inoculation. Furthermore, we tested the hypothesis whether the induction of ATGRXS13 by B. cinerea can inhibit the production of the phytoalexin camalexin, a major component of Arabidopsis defence against this fungus (Ferrari et al., 2003; Kliebenstein et al., 2005). PAD3 catalyzes the last step of camalexin biosynthesis and PAD3 expression correlates with camalexin production in response to B. cinerea (Schuhegger et al., 2006). The expression of $P A D 3$ and the accumulation of camalexin were similar in WT and grxs 13-1 plant inoculated with $B$. cinerea (Figure S6c,d). These results indicate that the induction of such classical defence genes or camalexin can not explain the resistance to $B$. cinerea observed in grxs 13 .

\section{DISCUSSION}

Hormones such as SA and JA are key regulators of the plant defence machinery. During plant infection by pathogens, these hormones control a highly complex network of sig- nalling cascades that lead to gene expression and improved resistance. SA is mainly associated with resistance to biotrophic pathogens whereas JA is associated with resistance against necrotrophic pathogens such as $B$. cinerea (Glazebrook, 2005). Here we show that infection of Arabidopsis by $B$. cinerea led to an increase in the level of both JA and SA detected at 36 hpi (Figure 1). Interestingly, a lack of JA production in the allene oxide synthase mutant dde2-2 (von Malek et al., 2002) resulted in a strong increase in SA accumulation and PR1 gene expression (Figures 1 and S1a). Furthermore, this mutant is hyper-susceptible to $B$. cinerea (Figure S1b). However, in sid2-1 plants, that do not accumulate $\mathrm{SA}$ in response to $B$. cinerea, the level of JA is similar to that in WT plants. These results indicate the existence of a negative effect of JA on the production of SA and SA-dependent events in Arabidopsis plants infected with B. cinerea. Although important, the repressive effect of JA on SA accumulation in WT plants is not fully efficient as SA accumulated and $P R 1$ was expressed (Figures 1 and S5).

Virulent pathogens can infect and invade plants despite attempted defence mechanisms deployed by their hosts. JA is a key regulator of basal plant defence against necrotrophic pathogens (Glazebrook, 2005) and it is produced in response to $B$. cinerea (Figure 1). Furthermore, plants affected in JA responses are more susceptible to necrotrophic fungi including B. cinerea (Figure S1b; Ferrari et al., 2003; Rowe and Kliebenstein, 2010; Thomma et al., 1998; Yang et al., 2007). We have identified a small number of genes, including the putative glutaredoxin ATGRXS13 presented here, that are repressed by JA and yet induced by $B$. cinerea. We postulate that such genes could potentially interfere with the metabolism of the plant and favor growth of the pathogen in planta. Supporting our assumption, mutations in ATGRXS13 resulted in an increased resistance to $B$. cinerea strain BMM. Both T-DNA mutants grxs13-1 and grxs13-2 exhibited reduced macroscopic symptoms of infection and a reduced pathogen growth (Figure 5). Therefore, it should be noted that there is a great diversity in $B$. cinerea strain virulence (Rowe and Kliebenstein, 2010) and we can not rule out the possibility that the phenotype we observed could be different with other $B$. cinerea strains. Glutaredoxins are small redox proteins of the thioredoxin superfamily the major functions of which are to reduce intra- and intermolecular disulfide bridges of target proteins or to reduce glutathionylated proteins. They can also serve as scaffold proteins for the assembly and delivery of [2Fe-2S] clusters most likely independently of their thiol-reductase activity (Rouhier et al., 2004, 2010; Bandyopadhyay et al., 2008). In plants, the role of GRX has mainly been studied in vitro and some publications indicate a role in the response to oxidative stress in vivo (Cheng et al., 2006) or in the development of petals and anthers (Xing et al., 2005; Xing and Zachgo, 2008). Recent studies have suggested an involvement of these proteins in plant defence. Over-expression of 
ATGRXC7/ROXY1 in Arabidopsis enhanced susceptibility to $B$. cinerea that correlates with an increased oxidative stress (Wang et al., 2009). However, over-expression of ATGRXS13 splice variants does not result in an increased oxidative stress (Figure S4). Furthermore, ATGRXC9/GRX480 has been shown to suppress the JA-responsive PDF1.2 expression (Ndamukong et al., 2007). We show now that
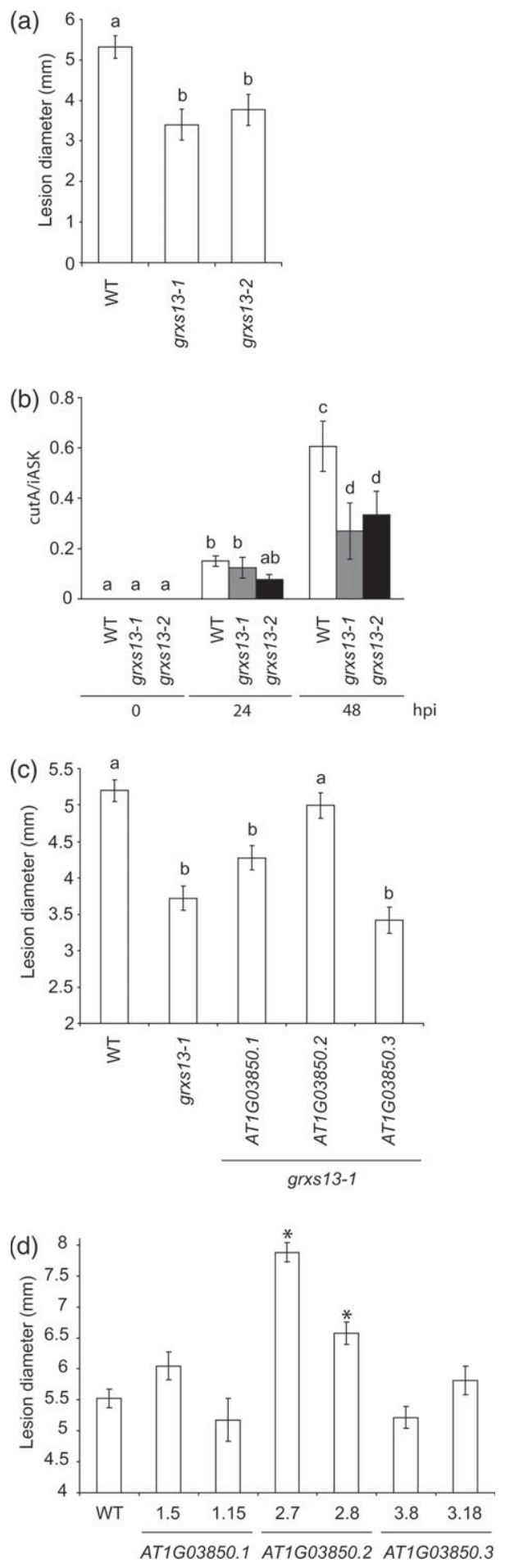

Figure 5. Phenotype of the grxs-13 mutants infected with $B$. cinerea and role of ATGRXS13 splice variants in Arabidopsis plants infected by $B$. cinerea. (a) Lesion diameter observed on wild-type (WT) Col and grxs13 mutants 3 days after inoculation with $B$. cinerea. The data represent the mean lesion diameters from 12 (grxs 13-1) and six (grxs13-2) independent experiments. In each experiment, 12-16 plants per genotype were infected with $B$. cinerea. Different letters represent groups which were significantly different from one another as determined by a one-way analysis of variance (ANOVA) followed by a multiple comparison with the Student-Newman-Keuls method $(P<0.05)$. (b) Fungal growth quantification on WT Col and grxs 13 mutants infected with $B$. cinerea. The abundance of genomic DNA of $B$. cinerea and $A$. thaliana was estimated by the quantification of CUTINASE-A gene from $B$. cinerea and IASK gene from $A$. thaliana and represented by the CUTA/IASK ratios. In each experiment, eight plants per genotype were infected with $B$. cinerea and the data are the mean of three independent experiments $( \pm S D)$. Different letters represent groups which were significantly different from one another as determined by a one-way ANOVA followed by a multiple comparison with the Student-Newman-Keuls method $(P<0.05)$.

(c) Infection phenotype of grxs13-1 mutant complemented with AtGRXS13 splice variants under the control of ATGRXS13 native promoter. In each experiment, sixteen plants per genotype were infected with $B$. cinerea and lesion diameters were measured after 3 days. The means of lesion diameter data from five independent experiments are shown $( \pm S D)$. Different letters represent groups which were significantly different from one another as determined by a Kruskal-Wallis one-way ANOVA on ranks followed by a multiple comparison with the Dunn's method. $(P<0.01)$.

(d) Infection phenotype of WT plants over-expressing ATGRXS13 splice variants. In each experiment, sixteen plants per genotype were infected with $B$. cinerea and lesion diameter was measured after 3 days. Two lines overexpressing AT1G03850.1 (1.5; 1.15), AT1G03850.2 (2.7; 2.8) and AT1G03850.3 $(3.8 ; 3.18)$ were tested. The means of lesion diameter data from three independent experiments are shown $( \pm S D)$. Significant differences $\left({ }^{*}\right)$ from WT plant were determined by a Kruskal-Wallis one-way ANOVA on ranks followed by a comparison with the Dunn's method $\left({ }^{*} P<0.01\right)$.

ATGRXS13, another glutaredoxin, is required for a successful colonization of Arabidopsis by the necrotrophic fungus Botrytis cinerea.

GRX from photosynthetic organisms are grouped into six different classes based on sequence identity of both the active site and a conserved motif involved in glutathione (GSH)-binding. Based on protein homology sequence, ATGRXS13 belongs to the class III (CCXx active site) that is specific to terrestrial plants (Couturier et al., 2009). However, no typical glutaredoxin activity has been shown for this protein so far. ATGRXS13 gene is induced and alternatively spliced into three different mRNAs (Figures 2, 3 and S2) in plants infected with B. cinerea. Only the AtGRXS13.2 cDNA sequence under the control of the native $A T G R X S 13$ promoter was able to complement the mutant grxs13-1 resulting in normal susceptibility to B. cinerea (Figure $5 \mathrm{c}$ ). This is further supported by our data showing that over-expression of ATGRXS13.2 but not of ATGRXS13.1 and ATGRXS13.3 in WT plants resulted in a greater susceptibility to $B$. cinerea (Figure $5 \mathrm{~d}$ ). Finally, ATGRXS13.2 exhibited the highest amount of transcripts after fungal infection compared with the other splice variants (Figure 3). Thus, only AtGRXS13.2 is likely to be crucial during the colonization of Arabidopsis by $B$. cinerea. Whereas the three predicted glutaredoxin proteins 


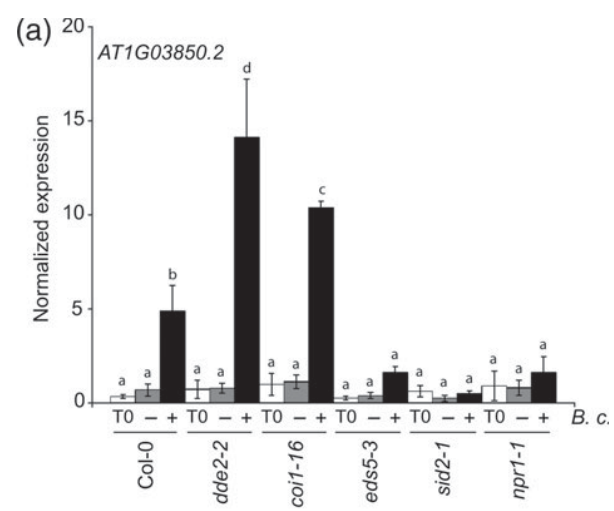

(b) ${ }^{10}$ AT1G03850.2

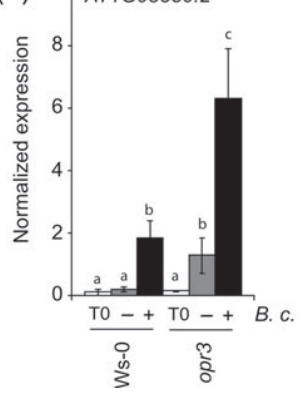

Figure 6. ATGRXS13.2 mRNA accumulation in various signalling mutants infected with $B$. cinerea.

The leaves from sixteen plants were not sprayed (white bars), sprayed with mock solution (grey bars) or B. cinerea spores (black bars) and were harvested after $24 \mathrm{~h}$. AT1G03850.2 transcript levels were quantified by realtime polymerase chain reaction (PCR) and expressed as normalized expression (no unit) to the plant reference gene AT4G26410 transcript level (Czechowski et al., 2005). Data represent the mean of three independent experiments $( \pm S D)$ and different letters represent groups which were significantly different from one another as determined by a one-way analysis of variance (ANOVA) followed by a multiple comparison with the StudentNewman-Keuls method $(P<0.05)$.

have the same $\mathrm{N}$-terminal sequence and the same putative catalytic site CCLG, only ATGRXS13.1 and ATGRXS13.2 display the typical thioredoxin fold characteristic of GRX (Figure 2b; Martin, 1995). Furthermore, the putative glutathione binding site is missing in AtGRXS13.3 (Figure 2b). Therefore, ATGRXS13.3 is unlikely to encode a functional GRX. The C-terminal part of the three isoforms is different and only ATGRXS13.2 contains the ALWL motif that is shared by many group III glutaredoxins from plants (Figure $2 b$ ). This motif is essential for the protein function of ATGRXC7 (ROXY1) during petal development (Li et al., 2009). Furthermore, only glutaredoxins possessing the ALWL motif can complement the altered petal development phenotype of roxy1-2 mutant (Li et al., 2009). Thus, our results show that during infection of $A$. thaliana the expression of what appears to be the most active splice variant of the ATGRXS13 gene is induced and required by $B$. cinerea for optimal infection.
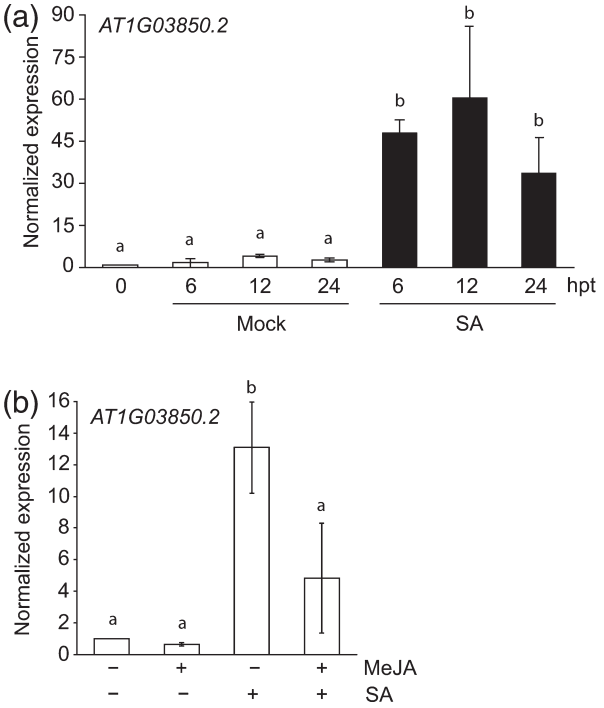

Figure 7. ATGRXS13.2 mRNA accumulation in wild-type (WT) plants treated with SA and MeJA.

(a) Accumulation of ATGRXS13.2 mRNA in response to salicylic acid (SA) treatment. The leaves from 10 plants were sprayed with Silwet-L77 $0.015 \%$ (white bars) or with $1 \mathrm{~mm}$ of SA (black bars) and harvested at the indicated time points.

(b) SA-induced accumulation of ATGRXS13.2 mRNA in plants pre-treated with MeJA. The leaves from 10 plants were sprayed with $\mathrm{EtOH}$ or $100 \mu \mathrm{M}$ of MeJA and sprayed $3 \mathrm{~h}$ later with Silwet-L77 $0.015 \%$ or $1 \mathrm{~mm}$ of SA. Leaves were harvested $6 \mathrm{~h}$ after SA treatment. For (a) and (b), AT1G03850.2 transcript levels were quantified by real-time polymerase chain reaction (PCR). Data are the mean of three independent experiments and are expressed as normalized expression (no unit) to the plant reference gene AT4G26410 transcript level (Czechowski et al., 2005). Different letters represent groups which were significantly different from one another as determined by a one-way analysis of variance (ANOVA) followed by a multiple comparison with the StudentNewman-Keuls method $(P<0.05)$. hpt: hour post treatment.

AT1G03850 was negatively controlled by JA-dependent pathways during infection but positively regulated by NPR1- and SA-dependent pathways as shown using various mutants (Figure 6). These data were confirmed by ectopic treatments of WT plants with SA and JA. SA induced the expression of ATGRXS13 and application of MeJA before SA resulted in a decreased expression of ATGRXS13 compared with a treatment with SA alone (Figure 7). These results indicate that the induction of ATGRXS13 is under the control of both hormones. Thus, our results indicate that JA produced after $B$. cinerea infection negatively controls the production of $S A$ and the expression of the SA-responsive gene ATGRXS13. While most reports on the SA/JA antagonism have documented a negative interference of SA on JA-dependent responses, some studies also reported a negative crosstalk of JA on SA-mediated signalling (Bostock, 2005; Koornneef and Pieterse, 2008). For instance, SA-dependent responses are not expressed in the cev1 mutant that constitutively activates JA responses (Ellis et al., 2002a,b). Plants mutated in COI1, a central regulator of the JA signalling, 


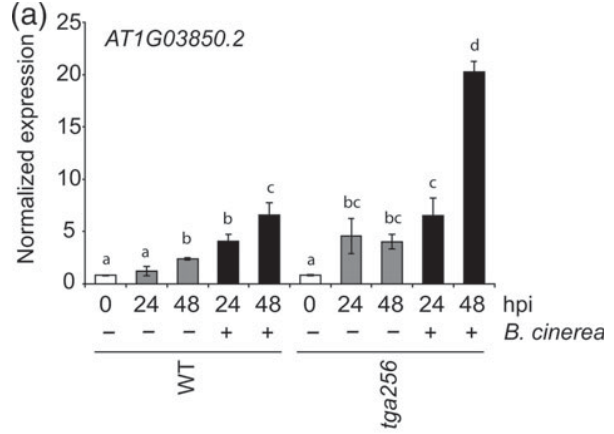

(b)

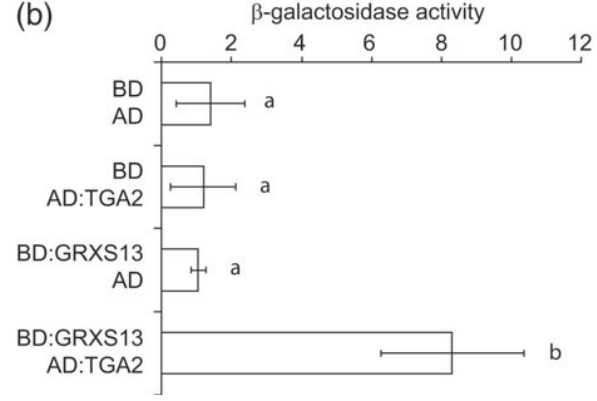

Figure 8. Relationship between ATGRXS13.2 and TGA

(a) AT1G03850.2 mRNA accumulation in tga256 triple mutant infected with $B$. cinerea. The leaves from sixteen plants were not sprayed (white bars), sprayed with mock solution (grey bars) or $B$. cinerea spores (black bars) and were harvested at the indicated time points. AT1G03850.2 transcript levels were quantified by real-time polymerase chain reaction (PCR) and expressed as normalized expression (no unit) to the plant reference gene AT4G26410 transcript level (Czechowski et al., 2005). Data are the mean of three independent experiments.

(b) Analysis of the interaction of ATGRXS13 with TGA2 in a quantitative yeast two-hybrid assay. The coding region of ATGRXS13 (AT1G03850.2) was fused to the GAL4 DNA-binding domain (BD:GRXS13), and that of TGA2 was fused to the GAL4 activation domain (AD:TGA2). After transformation of the respective plasmids in yeast MAV203 cells, $\beta$-galactosidase activity was measured. Three to seven independent clones were used for each construct in a single experiment. The results represent the average of three independent experiments.

For (a) and (b), different letters represent groups which were significantly different from one another as determined by a one-way analysis of variance (ANOVA) followed by a multiple comparison with the Student-Newman-Keuls method $(P<0.05)$

are more resistant to Pst DC3000 due to an elevated level of SA and SA-dependent defences (Kloek et al., 2001). Furthermore, the jin1 (jasmonate insensitive-1) mutant that is mutated in the ATMYC2 transcription factor also accumulates more SA after challenge with Pst DC3000 (Nickstadt et al., 2004). One may still wonder how relatively high levels of expression of ATGRX13 can be induced after $B$. cinerea infection despite the JA/SA crosstalk. A possible explanation might be that $B$. cinerea interferes with the existing crosstalk between JA and SA through the release of various pathogenicity factors, making possible that the crosstalk is not complete and would thus allow for some expression of ATGRXS13. Alternatively, such a pathogenicity factor might affect the repression of ATGRXS13 by JA. In summary, infection of Arabidopsis by $B$. cinerea leads to an SA-dependent increase in the expression of the second isoform of ATGRXS13 despite a negative crosstalk of JA on the SA-dependent responses. The details of the upregulation of $A T G R X S 13$ by $B$. cinerea in the general context of a negative interference by JA remain now to be elucidated.

The role of SA in the interaction between Arabidopsis and $B$. cinerea warrants a comment. We show that SA-dependent expression of ATGRXS13 is required for full infection by $B$. cinerea. The bik1 mutant affected in a membraneanchored kinase is more susceptible to $B$. cinerea due to an increased accumulation of SA (Veronese et al., 2006). Furthermore, the triple mutant wrky18wrky40wrky60 exhibits a higher susceptibility to $B$. cinerea that correlates with an enhanced expression of the SA marker PR1 (Xu et al., 2006). In contrast, some reports indicate that $S A$ is required for local resistance in Arabidopsis (Govrin and Levine, 2002; Ferrari et al., 2003) making it likely that SA has a dual role in the response to $B$. cinerea. When a low dose of SA (e.g. $0.5 \mathrm{~mm}$ ) is sprayed on Arabidopsis plants before inoculation, a greater susceptibility to $B$. cinerea is observed (Yang et al., 2007) whereas a high dose of SA (e.g. $5 \mathrm{~mm}$ ) induces resistance (Ferrari et al., 2003). These apparent discrepancies on the role of SA during the interaction between Arabidopsis and $B$. cinerea could also be explained by differences in experimental conditions and $B$. cinerea isolates used between studies as discussed recently by Rowe and Kliebenstein (2010).

Members of the TGA transcription factor family such as TGA2, TGA5 and TGA6 that act redundantly regulate SA-dependent defence responses (Zhang et al., 2003). It has been shown that these transcription factors contribute to basal resistance of Arabidopsis plants to B. cinerea. Thus, the triple mutant tga2tga5tga6 is more susceptible to the infection by this fungus (Zander et al., 2010). Here we show that ATGRXS13.2 over-accumulated in tga2tga5tga6 (Figure 8a) indicating that members of the TGA2 clade of transcription factors act as repressors of ATGRXS13 gene expression in response to $B$. cinerea. The presence of TGA binding elements in the sequence of ATGRXS13 promoter suggests that members of this TGA2 clade could directly bind to the promoter (Figure S5). However, we cannot rule out the possibility that this effect is indirect. It has been shown that the TGA2 clade is required for basal repression of SA-dependent gene expression such as PR1 in the absence of SA production (Zhang et al., 2003; Rochon et al., 2006). However, TGA2 was also shown to be an activator of SA-dependent gene expression (Zhang et al., 2003; Rochon et al., 2006). Based on our results, we propose a hypothetical model for the induction of ATGRXS13 and its role in plants infected with $B$. cinerea (Figure 9). JA-related mutants (in which SA over-accumulated, Figure 1) behave in the same way as the tga2tga5tga 6 mutant with regard to the 


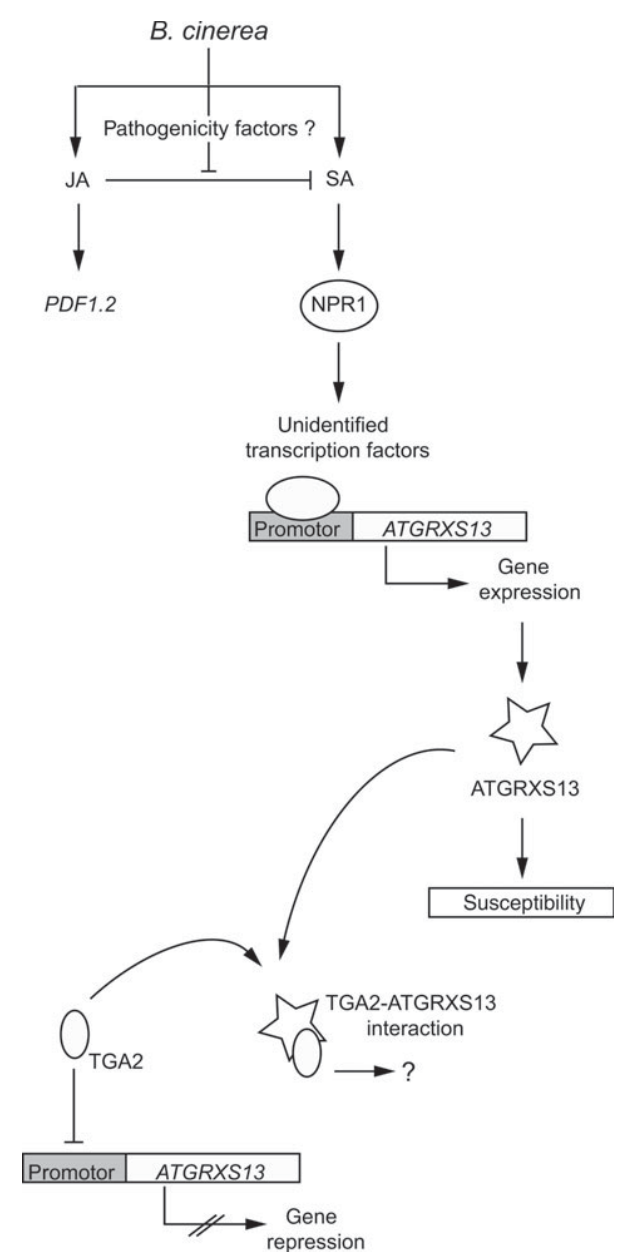

Figure 9. Hypothetical model of the regulation of ATGRXS13 during the infection of Arabidopsis by $B$. cinerea.

In plants infected with B. cinerea, ATGRXS13 is expressed and jasmonic acid (JA) and salicylic acid (SA) are produced. The repression of SA biosynthesis and SA-dependent gene expression by JA is indicated. However, this repression is only partial, SA is produced and the SA-dependent gene ATGRXS13 is expressed. B. cinerea might release some unknown pathogenicity factors that interfere with the crosstalk from JA to SA. ATGRXS13 expression is repressed by members of the TGA2 clade of transcription factors. During infection, ATGRXS13 expression is derepressed through a SAand NPR1-dependent pathway and some unidentified transcription factors can drive the expression of ATGRX13 that is required by $B$. cinerea to fully infect Arabidopsis plants. Finally, the interaction between ATGRXS13 and TGA2 suggests that ATGRXS13 could be involved in post-translational modification of TGA2 that would affect TGA2 activity.

expression of ATGRXS13 (Figures 6 and 8a). These data suggest that the enhanced expression of ATGRXS13 observed in JA-related mutants (Figure 6) can be the consequence of an overaccumulation of SA. In turn, SA could induce ATGRXS13 gene expression that was repressed by TGA2. We observed that the induction of ATGRXS13 is strongly impaired in SA-related mutants infected by $B$. cinerea (Figure 6). Thus, the expression of ATGRXS13 is negatively regulated by JA-dependent pathways in the absence of a stimulus such as an infection that stimulates SA accumulation. The triple tga mutant is deleted in the genes coding these three transcription factors (Li et al., 2001; Zhang et al., 2003). Thus, TGA2 is absent in this mutant but $A T G R X S 13$ is over-expressed in response to $B$. cinerea (Figure 8a). We can speculate that a positive regulation of ATGRXS13 expression by TGA2 is unlikely in plants infected by $B$. cinerea. Possibly, other transcription factors can positively regulate ATGRXS13 expression.

We have shown a direct interaction between ATGRXS13 and TGA2 suggesting that ATGRXS13 could be involved in post-translational modification of TGA2 that could affect TGA2 activity (Figures $8 b$ and 9). One of the consequences might be the induction of the expression of the ATGRXS13 gene. But the interaction between TGA2 and ATGRXS13 is unlikely to control $P R 1$ and $P D F 1.2$ expression in response to $B$. cinerea. Indeed, expression of both genes is unaffected by the grxs 13-1 mutation (Figure S6). Other reports highlighted an interaction of ATGRXs with TGA transcription factors in plants (Ndamukong et al., 2007; Li et al., 2009; Murmu et al., 2010). However, the mechanisms by which Arabidopsis glutaredoxin could regulate TGA transcription factors remains to be determined.

In conclusion, we have shown that virulence of $B$. cinerea on $A$. thaliana depends on the SA-dependent expression of a putative active splice variant of the glutaredoxin ATGRXS13 gene. This expression escapes a negative interference by $J A$ that is produced in response to $B$. cinerea infection. ATGRXS13 gene expression is also negatively regulated by TGA2 clade of transcription factors and both proteins can interact. These results illustrate and further our knowledge on the subtle changes mediated by virulent pathogens in their hosts that allow a successful colonization.

\section{EXPERIMENTAL PROCEDURES}

\section{Plant materials and growth conditions}

A. thaliana seeds were grown on a pasteurized soil composed of humus and perlite $(3: 1)$ in a growth chamber under a $12 \mathrm{~h}$ light/12 $\mathrm{h}$ dark cycle, $60-70 \%$ humidity, $16-18^{\circ} \mathrm{C}$ during the night and $20-22^{\circ} \mathrm{C}$ during the day. The ATGRXS13 mutant was grxs13-1 (SALK_046205) and grxs13-2 (SALK_088910).

\section{Pathogen infection}

B. cinerea strain BMM (Zimmerli et al., 2000) was grown on Difco potato dextrose agar (Becton Dickinson). Spores were harvested in water and filtered through glass wool and then diluted in quarterstrength Difco potato dextrose broth (PDB) for inoculation. For disease assays, $6 \mu \mathrm{L}$ of spore solution $\left(5 \times 10^{4}\right.$ spores $\left.\mathrm{mL}^{-1}\right)$ were deposited on three leaves of 5 weeks old plants. Lesion diameters were measured after 3 days. Fungal growth was measured as described previously (Gachon and Saindrenan, 2004). Plants were drop-inoculated and leaf discs were harvested at the indicated time points. For SA or JA measurements and total RNA isolation, plants were sprayed with spores $\left(2 \times 10^{5}\right.$ spores $\left.\mathrm{mL}^{-1}\right)$ and control plants with quarter-strength PDB. Entire leaves were harvested at the indicated time points and frozen in liquid nitrogen. The inoculated 
plants were kept under high humidity in a tray closed with a watersprayed transparent lid.

\section{Plant treatment}

Plants were sprayed with $1 \mathrm{~mm}$ of SA sodium salt (Sigma-Aldrich) dissolved in water containing $0.015 \%$ of Silwet L-77 (Koornneef et al., 2008). MeJA was dissolved as a $100 \mathrm{~mm}$ stock solution in absolute ethanol. Plants were sprayed with $100 \mu \mathrm{m}$ of MeJA diluted in water. Control plants were sprayed with water containing the same volume of Silwet L-77 or absolute ethanol. Entire leaves were harvested at the indicated time points and frozen in liquid nitrogen.

\section{Characterization of grxs 13 mutant alleles}

Plants homozygous for grxs13-1 and grxs13-2 mutations were identified by polymerase chain reaction (PCR) (Table S1). The positions of the T-DNA insertions were determined by DNA sequencing of the PCR products obtained with primers Lba1 and S13-1RP (for grxs 13-1), LBa1 and S13-2 RP or S13-2 LP (for grxs 13-2).

\section{RNA extraction and quantitative real-time PCR}

Total RNA was isolated using TRIzol reagent (Invitrogen, http:// www.invitrogen.com) and further used for retrotranscription into cDNA (Omniscript RT kit; Qiagen AG, http://www.qiagen.com). Quantitative real-time PCR was performed using Absolute QPCR SYBR Green Mix (ABgene, Thermo Fisher Scientific, http:// www.thermofisher.com). Gene expression values were normalized to the expression of the plant gene AT4G26410, previously described as a stable reference gene (Czechowski et al., 2005). The primers are listed in Table S1.

\section{Cloning procedures}

cDNA made from RNA extracted from B. cinerea-treated Arabidopsis plants were used to amplify ATGRXS13 splice variants by PCR (Phusion Taq Polymerase, Finzymes). Products were cloned into the pENTR-D-TOPO vector (Invitrogen), sequenced and LR-recombined with pB2GW7 (Karimi et al., 2002) to express ATGRXS13 splice variants under the control of the CaMV35S promoter. WT Col plants were transformed and two independent transgenic T2 plant lines per splice variant were selected for further infection experiments with $B$. cinerea. To obtain vectors in which ATGRXS13 splices variants were driven by ATGRXS13 native promoter, the $1500 \mathrm{bp}$ before the start codon of ATGRXS13 were amplified by PCR and then cloned into pDONRP4P1R (Invitrogen). A LR-recombination was performed using pENTR-D-TOPO containing ATGRXS13 splice variants, pDONRP4P1r and the destination vector pH7m24GW,3 (Karimi et al., 2002). Grxs13-1 mutants were transformed and one independent transgenic T2 plant line per splice variant was selected for further infection experiments with $B$. cinerea. The primers are listed in Table S1.

\section{Plant transformation}

Binary plasmids described above were electroporated into Agrobacterium tumefaciens strain GV3101 (pMp90) and selected on LB-agar plates containing gentamicin $\left(25 \mu \mathrm{g} \mathrm{mL}^{-1}\right)$, rifampicin $\left(50 \mu \mathrm{g} \mathrm{m}^{-1}\right)$ and spectinomycin $\left(100 \mu \mathrm{g} \mathrm{mL}^{-1}\right)$. Plants were transformed using the floral dipping method (Clough and Bent, 1998). Transgenic plants were selected either on glufosinate, hygromycin or by PCR.

\section{Yeast two-hybrid analysis}

The coding region of ATGRXS13.2 was amplified by PCR, cloned in pDONR223 and LR-recombined in the yeast vector pDEST-GBKT7
(Invitrogen). The TGA2 coding region (Ndamukong et al., 2007) was LR-recombined in the destination yeast vector pDEST-GAD. The activity of $\beta$-galactosidase was measured after transformation of the respective plasmids in yeast MAV203 cells.

\section{Camalexin, SA and JA quantification}

SA and camalexin were quantified as described by Nawrath and Métraux (1999) using $200 \mathrm{mg}$ of leaves. JA quantification was adapted from Schmelz et al., 2004 and Gundlach et al., 1992. About $500 \mathrm{mg}$ of leaf material was extracted twice with $1 \mathrm{ml}$ of extraction buffer (water/propan-1-ol/ $\mathrm{HCl}: 1 / 2 / 0.005, \mathrm{v} / \mathrm{v}$ ) at $70^{\circ} \mathrm{C}$ and using a homogenizer (Polytron, Kinematica) with $100 \mathrm{ng}$ of the internal standard dihydro-JA. Samples were mixed $15 \mathrm{sec}$ with $2 \mathrm{ml}$ of dichloromethane and centrifuged $2 \mathrm{~min}(10000 \mathrm{~g})$. The lower organic phase was dried by the addition of anhydrous $\mathrm{Na}_{2} \mathrm{SO}_{4}$. Carboxylic acids including JA were methylated $30 \mathrm{~min}$ to their corresponding methyl esters at room temperature by adding $20 \mu \mathrm{l}$ of $2 \mathrm{M}$ bis-trimethylsilyldiazomethane and $200 \mu \mathrm{l}$ of $\mathrm{MeOH}$. Methylation was stopped $30 \mathrm{~min}$ by adding $20 \mu \mathrm{l} 2 \mathrm{M}$ acetic acid (room temperature). Solvent was evaporated under a nitrogen stream and the sample was dissolved in $1 \mathrm{ml}$ of dichloromethane before loading onto a silica column (Chromabond; Macherey-Nagel AG, http:// www.mn-net.com) prewashed with hexane. After washing with hexane $(3 \times 2 \mathrm{ml})$, the column was eluted with $4 \mathrm{ml}$ of hexane/diethyl ether (2:1). The eluate was evaporated and dissolved in $25 \mu \mathrm{l}$ of hexane before its separation on a capillary column $(25 \mathrm{~m} \times 0.2 \mathrm{~mm})$ of BP-225 (SGE) fitted to a Hewlett Packard 5980GC coupled to a 5970 mass specific detector. The methyl esters of JA and dihydro-JA were detected by selective ion monitoring at $\mathrm{m} / \mathrm{z} 151$ and 156, respectively. JA amount (measured as methyl JA) was quantified by reference to the amount of internal standard. The results are expressed in $\mu \mathrm{g} \cdot \mathrm{g}^{-1} \mathrm{FW}$ of plant tissue.

\section{ACKNOWLEDGEMENTS}

We would like to thank all our colleagues for inspiring discussions. Linda Grainger is gratefully acknowledged for excellent technical help, Emmanuel Koen for help in statistical analysis and Dr. Antony Buchala for English editing. We thank John Browse and Christophe Ringli for providing the JA mutants opr3 and dde2-2. The CNRS, the Swiss NSF (grant 125370 for JPM) and the Deutsche Forschungsgemeinschaft are gratefully acknowledged for financial support.

\section{SUPPORTING INFORMATION}

Additional Supporting Information may be found in the online version of this article:

Figure S1. Responses of the allene oxide synthase mutant dde2-2 to B. cinerea.

Figure S2. Alignment of the genomic sequence of AT1G03850 to the cDNA of the three ATG1G03850 splice variants.

Figure S3. Phenotype of the grxs 13 mutants infected with B. cinerea. Figure S4. Histochemical detection of ROS accumulation by diaminobenzidine (DAB) staining in wild type Col plants and in plants over-expressing GRXS13 splice variants.

Figure S5. Sequence of the $1500 \mathrm{bp}$ upstream of the transcriptional starting site of ATGRXS13.

Figure S6. Role of ATGRXS13 in the accumulation of mRNA coding classical defence genes induced after $B$. cinerea infection and in the accumulation of the phytoalexin camalexin.

Table S1. List of primers used.

Please note: As a service to our authors and readers, this journal provides supporting information supplied by the authors. Such materials are peer-reviewed and may be re-organized for online delivery, but are not copy-edited or typeset. Technical support 
issues arising from supporting information (other than missing files) should be addressed to the authors.

\section{REFERENCES}

Alonso, J.M., Stepanova, A.N., Leisse, T.J. et al. (2003) Genome-wide insertional mutagenesis of Arabidopsis thaliana. Science, 301, 653-657.

Bandyopadhyay, S., Gama, F., Molina-Navarro, M.M. et al. (2008) Chloroplast monothiol glutaredoxins as scaffold proteins for the assembly and delivery of [2Fe-2S] clusters. EMBO J. 27, 1122-1133.

Bari, R. and Jones, J. (2009) Role of plant hormones in plant defence responses. Plant Mol. Biol. 69, 473-488.

Boller, T. and Felix, G. (2009) A renaissance of elicitors: perception of microbe-associated molecular patterns and danger signals by pattern-recognition receptors. Annu. Rev. Plant Biol. 60, 379-406.

Bostock, R.M. (2005) Signal crosstalk and induced resistance: straddling the line between cost and benefit. Annu. Rev. Phytopathol. 43, 545-580.

Chassot, C., Nawrath, C. and Métraux, J.P. (2007) Cuticular defects lead to full immunity to a major plant pathogen. Plant J. 49, 972-980.

Cheng, N.H., Liu, J.Z., Brock, A., Nelson, R.S. and Hirschi, K.D. (2006) AtGRXcp, an Arabidopsis chloroplastic glutaredoxin, is critical for protection against protein oxidative damage. J. Biol. Chem. 281, 26280-26288.

Chisholm, S.T., Coaker, G., Day, B. and Staskawicz, B.J. (2006) Host-microbe interactions: shaping the evolution of the plant immune response. Cell, 124, 803-814.

Clough, S.J. and Bent, A.F. (1998) Floral dip: a simplified method for Agrobacterium-mediated transformation of Arabidopsis thaliana. Plant J. 16 735-743.

Couturier, J., Jacquot, J.P. and Rouhier, N. (2009) Evolution and diversity of glutaredoxins in photosynthetic organisms. Cell. Mol. Life Sci. 66, 25392557.

da Cunha, L., Sreerekha, M.-V. and Mackey, D. (2007) Defense suppression by virulence effectors of bacterial phytopathogens. Curr. Opin. Plant Biol. 10, 349-357.

Czechowski, T., Stitt, M., Altmann, T., Udvardi, M.K. and Scheible, W.R. (2005) Genome-wide identification and testing of superior reference genes for transcript normalization in Arabidopsis. Plant Physiol. 139, 5-17.

Davis, E.L., Hussey, R.S., Mitchum, M.G. and Baum, T.J. (2008) Parasitism proteins in nematode-plant interactions. Curr. Opin. Plant Biol. 11, 360-366.

Durrant, W.E. and Dong, X. (2004) Systemic acquired resistance. Annu. Rev. Phytopathol. 42, 185-209.

Ellis, C., Karafyllidis, I., Wasternack, C. and Turner, J.G. (2002a) The Arabidopsis mutant cev1 links cell wall signaling to jasmonate and ethylene responses. Plant Cell, 14, 1557-1566.

Ellis, C., Karafyllidis, I. and Turner, J.G. (2002b) Constitutive activation of jasmonate signaling in an Arabidopsis mutant correlates with enhanced resistance to Erysiphe cichoracearum, Pseudomonas syringae, and Myzus persicae. Mol. Plant-Microbe Interact. 15, 1025-1030.

Ferrari, S., Plotnikova, J.M., De Lorenzo, G. and Ausubel, F.M. (2003) Arabidopsis local resistance to Botrytis cinerea involves salicylic acid and camalexin and requires EDS4 and PAD2, but not SID2, EDS5 or PAD4. Plant J. 35, 193-205.

Gachon, C. and Saindrenan, P. (2004) Real-time PCR monitoring of fungal development in Arabidopsis thaliana infected by Alternaria brassicicola and Botrytis cinerea. Plant Physiol. Biochem. 42, 367-371.

Garcion, C., Baltensperger, R., Fournier, T., Pasquier, J., Schnetzer, M.A., Gabriel, J.P. and Métraux, J.P. (2006) FiRe and microarrays: a fast answer to burning questions. Trends Plant Sci. 11, 320-322.

Glazebrook, J. (2005) Contrasting mechanisms of defense against biotrophic and necrotrophic pathogens. Annu. Rev. Phytopathol. 43, 205-227.

Gouet, P., Robert, X. and Courcelle, E. (2003) ESPript/ENDscript: extracting and rendering sequence and $3 \mathrm{D}$ information from atomic structures of proteins. Nucleic Acid Res. 31, 3320-3323.

Govrin, E.M. and Levine, A. (2002) Infection of Arabidopsis with a necrotrophic pathogen, Botrytis cinerea, elicits various defense responses but does not induce systemic acquired resistance (SAR). Plant Mol. Biol. 48, 267-276.

Gundlach, H., Muller, M.J., Kutchan, T.M. and Zenk, M.H. (1992) Jasmonic acid is a signal transducer in elicitor-induced plant cell cultures. Proc. Natl. Acad. Sci. USA, 89, 2389-2393.

Heck, S., Grau, T., Buchala, A., Métraux, J.P. and Nawrath, C. (2003) Genetic evidence that expression of NahG modifies defence pathways independent of salicylic acid biosynthesis in the Arabidopsis-Pseudomonas syringae pv. tomato interaction. Plant J. 36, 342-352.

Jones, D.T. (1999) Protein secondary structure prediction based on positionspecific scoring matrices. J. Mol. Biol. 292, 195-202.

Karimi, M., Inze, D. and Depicker, A. (2002) GATEWAY vectors for Agrobacterium-mediated plant transformation. Trends Plant Sci. 7, 193-195.

Kliebenstein, D.J., Rowe, H.C. and Denby, K.J. (2005) Secondary metabolites influence Arabidopsis/Botrytis interactions: variation in host production and pathogen sensitivity. Plant J. 44, 25-36.

Kloek, A.P., Verbsky, M.L., Sharma, S.B., Schoelz, J.E., Vogel, J., Klessig, D.F. and Kunkel, B.N. (2001) Resistance to Pseudomonas syringae conferred by an Arabidopsis thaliana coronatine-insensitive (coi1) mutation occurs through two distinct mechanisms. Plant J. 26, 509-522.

Koornneef, A. and Pieterse, C.M. (2008) Cross talk in defense signaling. Plant Physiol. 146, 839-844.

Koornneef, A., Leon-Reyes, A., Ritsema, T., Verhage, A., Den Otter, F.C., Van Loon, L.C. and Pieterse, C.M. (2008) Kinetics of salicylate-mediated suppression of jasmonate signaling reveal a role for redox modulation. Plant Physiol. 147, 1358-1368.

Li, X., Song, Y., Century, K., Straight, S., Ronald, P., Dong, X., Lassner, M. and Zhang, Y. (2001) A fast neutron deletion mutagenesis-based reverse genetics system for plants. Plant J. 27, 235-242.

Li, S., Lauri, A., Ziemann, M., Busch, A., Bhave, M. and Zachgo, S. (2009) Nuclear activity of ROXY1, a glutaredoxin interacting with TGA factors, is required for petal development in Arabidopsis thaliana. Plant Cell, 21, 429441.

von Malek, B., van der Graaff, E., Schneitz, K. and Keller, B. (2002) The Arabidopsis male-sterile mutant dde2-2 is defective in the ALLENE OXIDE SYNTHASE gene encoding one of the key enzymes of the jasmonic acid biosynthesis pathway. Planta, 216, 187-192.

Martin, J.L. (1995) Thioredoxin-a fold for all reasons. Structure, 3, 245-250.

Métraux, J.-P., Jackson, R.W., Esther, S. and Goldbach, R.W. (2009) Plant pathogens as suppressors of host defense. In Advances in Botanical Research (Van Loon, L.C., ed). Amsterdam: Elsevier Ltd, pp. 39-89.

Murmu, J., Bush, M.J., DeLong, C., Li, S., Xu, M., Khan, M., Malcolmson, C., Fobert, P.R., Zachgo, S. and Hepworth, S.R. (2010) Arabidopsis bZIP transcription factors TGA9 and TGA10 interact with floral glutaredoxins ROXY1 and ROXY2 and are redundantly required for anther development. Plant Physiol. 154, 1492-1504.

Nawrath, C. and Métraux, J.P. (1999) Salicylic acid induction-deficient mutants of Arabidopsis express PR-2 and PR- 5 and accumulate high levels of camalexin after pathogen inoculation. Plant Cell, 11, 1393-1404.

Ndamukong, I., Abdallat, A.A., Thurow, C., Fode, B., Zander, M., Weigel, R and Gatz, C. (2007) SA-inducible Arabidopsis glutaredoxin interacts with TGA factors and suppresses JA-responsive PDF1.2 transcription. Plant J. 50, 128-139.

Nickstadt, A., Thomma, B.P., Feussner, I., Kangasjärvi, J., Zeier, J., Loeffler, C., Scheel, D. and Berger, S. (2004) The jasmonate-insensitive mutant jin1 shows increased resistance to biotrophic as well as necrotrophic pathogens. Mol. Plant Pathol. 5, 425-434.

Nürnberger, T. and Kemmerling, B. (2009) PAMP-triggered basal immunity in plants. In Advances in Botanical Research, Vol. 51 (Van Loon, L.C., ed). Amsterdam: Elsevier Ltd, pp. 1-38.

Qin, X.F., Holuigue, L., Horvath, D.M. and Chua, N.H. (1994) Immediate early transcription activation by salicylic acid via the cauliflower mosaic virus as-1 element. Plant Cell, 6, 863-874.

Robert-Seilaniantz, A., Navarro, L., Bari, R. and Jones, J.D.G. (2007) Pathological hormone imbalances. Curr. Opin. Plant Biol. 10, 372-379.

Rochon, A., Boyle, P., Wignes, T., Fobert, P.R. and Despres, C. (2006) The coactivator function of Arabidopsis NPR1 requires the core of its $\mathrm{BTB} / \mathrm{POZ}$ domain and the oxidation of C-terminal cysteines. Plant Cell, 18, 36703685.

Rouhier, N., Gelhaye, E. and Jacquot, J.P. (2004) Plant glutaredoxins: still mysterious reducing systems. Cell. Mol. Life Sci. 61, 1266-1277.

Rouhier, N., Couturier, J., Johnson, M.K. and Jacquot, J.P. (2010) Glutaredoxins: roles in iron homeostasis. Trends Biochem. Sci. 35, 43-52.

Rowe, H.C. and Kliebenstein, D.J. (2010) All mold is not alike: the importance of intraspecific diversity in necrotrophic plant pathogens. PLoS Pathog. 6 e1000759.

Rowe, H.C., Walley, J.W., Corwin, J., Chan, E.K., Dehesh, K. and Kliebenstein, D.J. (2010) Deficiencies in jasmonate-mediated plant defense reveal 
quantitative variation in Botrytis cinerea pathogenesis. PLoS Pathog. 15, e1000861.

Schmelz, E.A., Engelberth, J., Tumlinson, J.H., Block, A. and Alborn, H.T. (2004) The use of vapor phase extraction in metabolic profiling of phytohormones and other metabolites. Plant J. 39, 790-808.

Schuhegger, R., Nafisi, M., Mansourova, M., Petersen, B.L., Olsen, C.E., Svatos, A., Halkier, B.A. and Glawischnig, E. (2006) CYP71B15 (PAD3) catalyzes the final step in camalexin biosynthesis. Plant Physiol. 141, 1248-1254.

Spoel, S.H., Koornneef, A., Claessens, S.M.C. et al. (2003) NPR1 modulates crosstalk between salicylate- and jasmonate-dependent defense pathways through a novel function in the cytosol. Plant Cell, 15, 760-770.

Stintzi, A. and Browse, J. (2000) The Arabidopsis male-sterile mutant, opr3, lacks the 12-oxophytodienoic acid reductase required for jasmonate synthesis. Proc. Natl Acad. Sci. USA, 97, 10625-10630.

Thomma, B.P.H.J., Eggermont, K., Penninckx, I.A.M.A., Mauch-Mani, B., Vogelsang, R., Cammue, B.P.A. and Broekaert, W.F. (1998) Separate jasmonate-dependent and salicylate-dependent defense- response pathways in Arabidopsis are essential for resistance to distinct microbial pathogens. Proc. Natl Acad. Sci. USA, 95, 15107-15111.

Thompson, J.D., Higgins, D.G. and Gibson, T.J. (1994) CLUSTAL W: improving the sensitivity of progressive multiple sequence alignment through sequence weighting, position-specific gap penalties and weight matrix choice. Nucleic Acids Res. 22, 4673-4680.

Van Etten, H., Temporini, E. and Wasmann, C. (2001) Phytoalexin (and phytoanticipin) tolerance as a virulence trait: why is it not required by all pathogens? Physiol. Mol. Plant Pathol. 59, 83-93.

Van Loon, L.C., Bakker, P.A.H.M. and Pieterse, C.M.J. (1998) Systemic resistance induced by rhizosphere bacteria. Annu. Rev. Phytopathol. 36 453-483.

Veronese, P., Nakagami, H., Bluhm, B., AbuQamar, S., Chen, X., Salmeron, J., Dietrich, R.A., Hirt, H. and Mengiste, T. (2006) The membrane-anchored BOTRYTIS-INDUCED KINASE1 plays distinct roles in Arabidopsis resistance to necrotrophic and biotrophic pathogens. Plant Cell, 18, 257-273.
Vlot, A.C., Klessig, D.F. and Park, S.-W. (2008) Systemic acquired resistance: the elusive signal(s). Curr. Opin. Plant Biol. 11, 436-442.

Wang, Z., Xing, S., Birkenbihl, R.P. and Zachgo, S. (2009) Conserved functions of Arabidopsis and rice CC-type glutaredoxins in flower development and pathogen response. Mol. Plant. 2, 323-335.

Xie, D.X., Feys, B.F., James, S., Nieto-Rostro, M. and Turner, J.G. (1998) COI1: an Arabidopsis gene required for jasmonate-regulated defense and fertility. Science, 280, 1091-1094.

Xing, S. and Zachgo, S. (2008) ROXY1 and ROXY2, two Arabidopsis glutaredoxin genes, are required for anther development. Plant J. 53, 790801.

Xing, S., Rosso, M.G. and Zachgo, S. (2005) ROXY1, a member of the plant glutaredoxin family, is required for petal development in Arabidopsis thaliana. Development, 132, 1555-1565.

Xu, X., Chen, C., Fan, B. and Chen, Z. (2006) Physical and functional interactions between pathogen-induced Arabidopsis WRKY18, WRKY40, and WRKY60 transcription factors. Plant Cell, 18, 1310-1326.

Yang, W., Devaiah, S.P., Pan, X., Isaac, G., Welti, R. and Wang, X. (2007) AtPLAl is an acyl hydrolase involved in basal jasmonic acid production and Arabidopsis resistance to Botrytis cinerea. J. Biol. Chem. 282, 1811618128.

Zander, M., Camera, S.L., Lamotte, O., Métraux, J.-.P. and Gatz, C. (2010) Arabidopsis thaliana class-II TGA transcription factors are essential activators of jasmonic acid/ethylene-induced defense responses. Plant J. 61 200-210.

Zhang, Y.L., Tessaro, M.J., Lassner, M. and Li, X. (2003) Knockout analysis of Arabidopsis transcription factors TGA2, TGA5, and TGA6 reveals their redundant and essential roles in systemic acquired resistance. Plant Cell, 15, 2647-2653.

Zimmerli, L., Jakab, C., Métraux, J.P. and Mauch-Mani, B. (2000) Potentiation of pathogen-specific defense mechanisms in Arabidopsis by beta-aminobutyric acid. Proc. Natl Acad. Sci. USA, 97, 12920-12925. 